\title{
Lesson of the month 1: Lobar pulmonary consolidation in an immunocompromised host
}

\author{
Authors: Daniel ] Reynolds, ${ }^{\mathrm{A}}$ Carl A Andersen, ${ }^{\mathrm{A}}$ Sumedh S Hoskote, ${ }^{\mathrm{B}}$ Hee Eun Lee, ${ }^{\mathrm{C}}$ Aditya Raghunathan, ${ }^{\mathrm{D}}$ \\ Sanjay Kalra ${ }^{B}$ and Andrew $\mathrm{H}$ Limper $^{B}$
}

\begin{abstract}
A 19-year-old male with a history of idiopathic panuveitis, currently taking methotrexate and infliximab, presented to our institution with 6 weeks of cough, dyspnoea and fevers. He had failed outpatient antimicrobial therapy. Computerised tomography (CT) of the chest revealed the presence of a lobar pneumonia and he was treated with broad spectrum antibiotics, which did not improve his symptoms. Bronchoalveolar lavage was performed with a transbronchial lung biopsy because of the diagnostic uncertainty of the patient's presentation. Pathology revealed non-budding yeasts, consistent with Pneumocystis. Serological and urine studies were positive for both Histoplasma and Blastomyces. The diagnosis of Histoplasma pneumonia was made because of the presentation being inconsistent with Pneumocystis pneumonia, and serology, urine and pathology testing being more consistent with Histoplasma. The patient was treated with oral itraconazole and was doing well at follow-up 12 weeks after hospitalisation.
\end{abstract}

KEYWORDS: Blastomyces, fungi, Histoplasma, immunosuppression, Pneumocystis, pneumonia

\section{Case presentation}

A 19-year-old male with idiopathic panuveitis diagnosed 5 years previously, which was initially treated with prednisone and then with infliximab along with topical therapy, presented to the clinic with 6 weeks of cough, greenish sputum, left-sided chest pain, haemoptysis, exertional dysponea, fevers, chills and night sweats. His primary doctor initially treated him with amoxicillin-clavulanate but his symptoms persisted and he was referred to our institution for admission.

\section{Assessment}

At admission, the patient was febrile $\left(38.4^{\circ} \mathrm{C}\right)$ and tachycardic (109 bpm). Other than bilateral scattered crackles, the physical

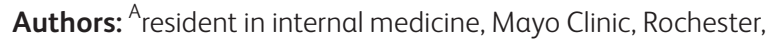
USA; ${ }^{B}$ consultant in pulmonary and critical care medicine, Mayo Clinic, Rochester, USA; ' resident in laboratory medicine, Mayo Clinic, Rochester, USA; ${ }^{D}$ Xconsultant in laboratory medicine and pathology, Mayo Clinic, Rochester, USA examination was normal. Laboratory testing showed a normal leukocyte count $(7,100 / \mu \mathrm{L})$, mildly elevated C-reactive protein $(8.2 \mathrm{mg} / \mathrm{L})$ and an elevated angiotensin converting enzyme (ACE) level (120 U/L; normal 8-53 U/L).

The patient was a college student living in a dormitory. He denied using recreational drugs, alcohol or tobacco. He had lived in Iowa for his entire life. He denied travel to a tuberculosis-endemic region, camping or hiking. He often visited a friend who had a pet dog and cat. He was not receiving Pneumocystis pneumonia prophylaxis.

A computerised tomography (CT) scan of the chest revealed consolidation in the lingula with additional bilateral micronodularity and hilar/mediastinal lymphadenopathy (Fig 1). Broad-spectrum coverage was provided with vancomycin and levofloxacin. An extensive diagnostic work-up was pursued, including serum and urine studies, bronchoalveolar lavage (BAL) and transbronchial lung biopsy (Table 1). BAL cell differential revealed lymphocytosis (42\%) with no malignant cells. Serological and urine antigen studies demonstrated reactivity for Blastomyces and Histoplasma. The lung biopsy showed necrotising granulomatous inflammation with scattered, non-budding degenerative yeasts $(2-4 \mu \mathrm{m})$, suggestive of Pneumocystis species (Fig 2).

\section{Diagnosis}

There was considerable diagnostic uncertainty with evidence for several infectious causes, including Pneumocystis, Blastomyces and Histoplasma. Furthermore, lymphocytic alveolitis (BAL lymphocytes $>20 \%$ ) can be present in various non-infectious conditions, including sarcoidosis, hypersensitivity pneumonitis, drug-induced lung disease, pulmonary lymphomas and lymphoproliferative disorders.

The possibility of sarcoidosis was entertained, as it would explain the panuveitis, and may also be manifesting in the lungs. Further, sarcoid-like granulomatous reactions have been reported with infliximab and other anti-tumour necrosis factor agents. ${ }^{1}$ Although granulomatous inflammation was seen on biopsy, the presence of fungal elements suggested infection. Lobar consolidation is unusual for sarcoidosis, which usually presents as hilar/mediastinal lymphadenopathy and/or multiple nodules in a perilymphatic distribution. ACE elevation lacks specificity as it can also be seen in fungal or 
Fig 1. Computerised tomography of the chest at the time of admission. Consolidation is noted in the lingula of the left upper lobe on lung (A) and mediastinal (B) windows. Parenchymal micronodularity is present bilaterally (A and $\mathrm{C}$ ) and hilar and mediastinal lymphadenopathy is seen in B and $\mathrm{D}$.
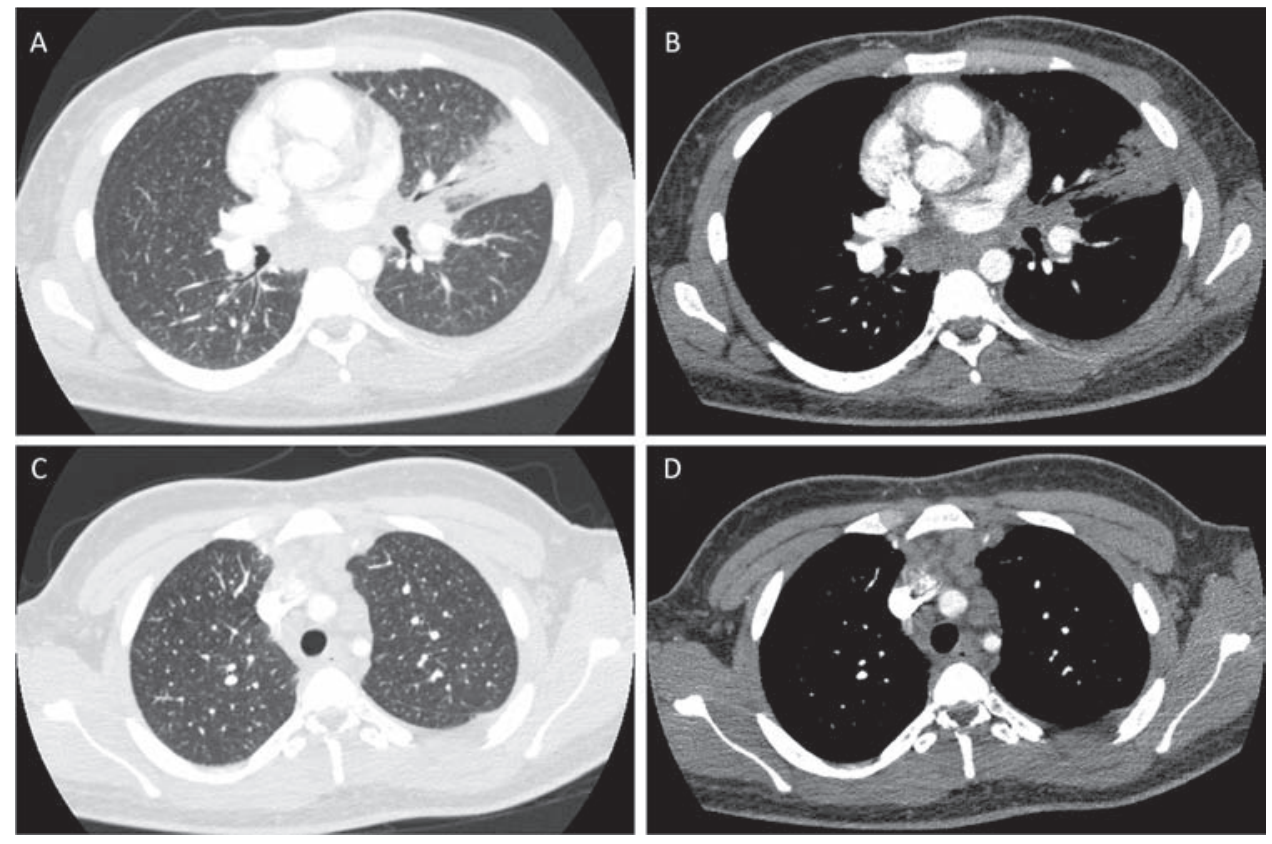

mycobacterial infection, diabetes, hypothyroidism, and other conditions.

The differential diagnosis was narrowed to the three most likely fungal infections, namely Pneumocystis pneumonia, blastomycosis and histoplasmosis. While imaging can raise suspicion for Pneumocystis infection, the diagnosis is confirmed by analysing sputum, BAL or histology. Pneumocystis pneumonia can produce a variety of radiographic appearances, including patchy upper-lobe-predominant ground glass opacities, and cystic lesions; $;^{2,3}$ but lobar consolidation is unusual. In non-HIV-infected immunocompromised patients, polymerase chain reaction (PCR) has excellent sensitivity and negative predictive value from induced sputum $(100 \% ; 100 \%)$ and BAL ( $84 \%$; $98.3 \%)$ samples. ${ }^{4}$ However, the histologic findings suggesting Pneumocystis were challenging to interpret in the context of negative PCR results for this organism.

On histology, Pneumocystis jirovecii shares many morphologic similarities with Histoplasma capsulatum. Both are thin-walled yeasts measuring $2-8 \mu \mathrm{m}$ in size, appearing collapsed or distorted in tissue sections. Pneumocystis often exhibits two larger intracystic bodies on silver stain and lacks budding forms. In contrast, Histoplasma has a smaller capsular dot and multiplies by budding. ${ }^{5}$ When present, budding forms can suggest Histoplasma; however, their absence does not exclude this possibility. ${ }^{5}$

In this case, the yeasts did not show budding, a capsular dot or intracystic bodies. Although granulomatous inflammation is atypical for Pneumocystis, it has been reported in $4-5 \%$ of patients. ${ }^{5}$ Unfortunately, the degenerative nature of yeast forms,
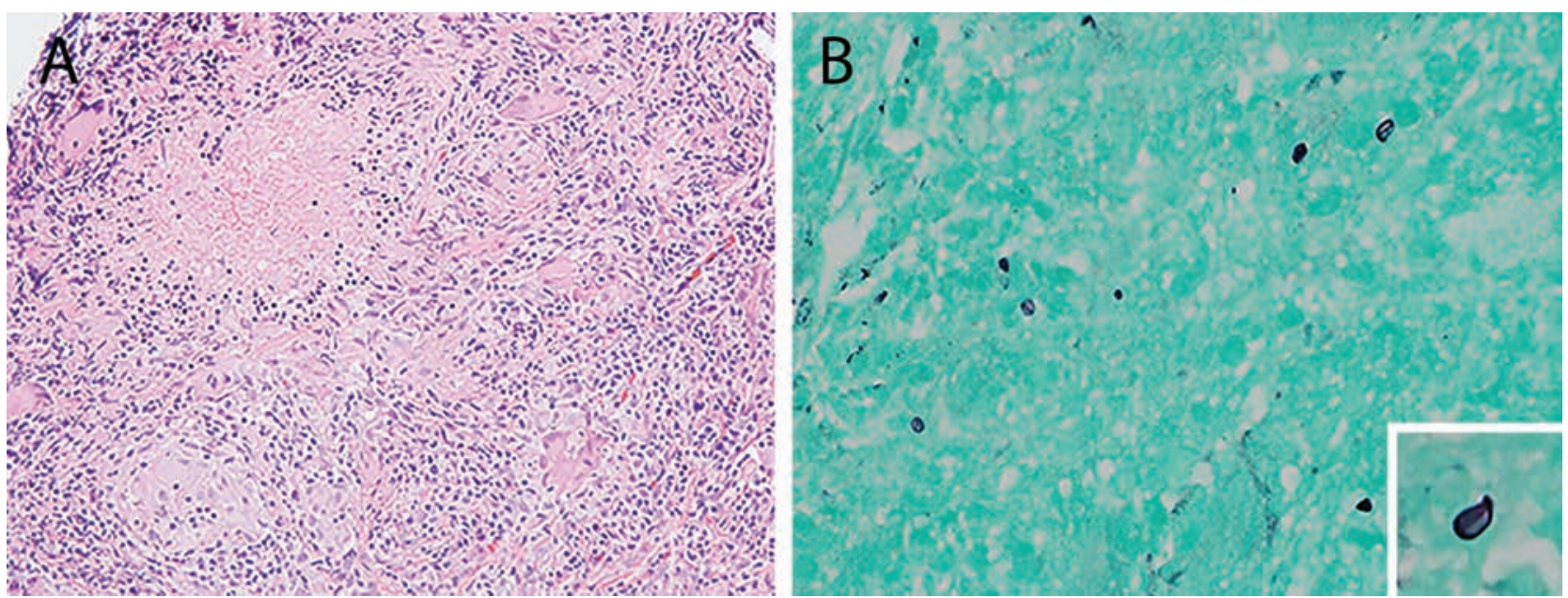

Fig 2. Photomicrographs from transbronchial lung biopsy. A - granulomatous inflammation with necrosis (hematoxylin and eosin; original magnification x200); $B$ - a few thin-walled non-budding degenerative yeasts $(2-5 \mu \mathrm{m})$ are sparsely found in the necrotising area (Gomori methenamine silver stain, original magnification $\times 1000$ ). 
Table 1. Summary of microbiologic data obtained by hospital day 2

\section{Test}

Aspergillus antigen, serum

Blastomyces antibody, serum

Blastomyces antigen, urine*

Coccidioides IgG, serum

Coccidioides IgM, serum

Coccidioides complement fixation

Cryptococcus antigen, serum

Histoplasma - mycelial, serum

Histoplasma - yeast, serum

Histoplasma - immunodiffusion, serum

Histoplasma antigen, urine

Sporothrix antibody, serum

HIV $1 / 2$ antigen and antibody screen, serum $M$ tuberculosis QuantiFERON ${ }^{\mathrm{TM}}$, blood

$M$ pneumoniae IgG, serum

$M$ pneumoniae IgM, serum

$Q$ fever IgG, serum

Q fever IgM, serum

Coxiella burnetti PCR, serum

Cell differential, BAL

> Alveolar macrophages

$>$ Lymphocytes

$>$ Neutrophils

AFB stain, BAL

Nocardia stain, BAL

Fungal smear, BAL

Bacterial culture, BAL

Adenovirus PCR, BAL

$M$ tuberculosis PCR, BAL

Influenza $A$ and $B$ PCR, BAL

Respiratory syncytial virus PCR, BAL

Legionella PCR, BAL

Pneumocystis PCR, BAL

Aspergillus antigen, $\mathrm{BAL}$

Pneumocystis PCR, sputum

M tuberculosis PCR, sputum

AFB stain, sputum

Bacterial culture, sputum

$S$ aureus PCR, nasal swab

$\mathrm{AFB}=$ acid-fast bacillus; $\mathrm{BAL}=$ bronchoalveolar lavage; $\mathrm{PCR}=$ polymerase chain reaction

*Test performed by MiraVista Diagnostics, Indianapolis, IN, USA and their sparse dispersion on a necrotic background, made definitive identification challenging on microscopy.

Blastomyces and Histoplasma are dimorphic fungi, existing as yeasts at body temperature and hyphae at relatively cooler temperatures $\left(<35^{\circ} \mathrm{C}\right)$. Blastomycosis and histoplasmosis are typically diagnosed by fungal stain, cytology, serology or urine/ serum antigen detection. Fungal cultures take several weeks to grow and are highly specific, but are often not available early in the evaluation and, therefore, are not helpful in making the initial diagnosis. Histoplasma PCR, a recent development, has $100 \%$ specificity for BAL samples.

Blastomyces antigen detection in urine or serum is positive in $92 \%$ of patients with disseminated or pulmonary infections. ${ }^{6}$ We considered blastomycosis based on positive urine antigen testing. However, Blastomyces antigen tests are known to crossreact with Histoplasma antigen. Furthermore, Blastomyces has a large $(10-15 \mu \mathrm{m})$ yeast form with broad-based buds and refractile walls, and was unlikely based on pathology.

Histoplasmosis was suspected in this patient with positive Histoplasma urine antigen as well as serology. Histoplasma serology is positive in $90 \%$ of subacute presentations. ${ }^{6}$ Serologic testing consists of complement fixation (yeast and mycelial antigens), and immunodiffusion ( $\mathrm{H}$ and $\mathrm{M}$ precipitins). A single titre $\geq 1: 32$ or a fourfold rise for either yeast or mycelial antibodies is considered diagnostic. ${ }^{7}$ Eventually, in our patient, histoplasmosis was confirmed on BAL and sputum culture in 4 weeks.

\section{Management}

Management recommendations for histoplasmosis are presented in Table $2 .{ }^{8}$ Our patient underwent treatment with itraconazole $200 \mathrm{mg}$ twice daily, with plans to continue this for the duration of his immunosuppression. His symptoms resolved within 1 month of therapy and radiographic infiltrates were seen to be resolving at 6 weeks; he continued to do well at 12-week follow-up.

Azole antifungals have significant drug-drug interactions via the cytochrome P450 system so therapeutic drug monitoring is essential. Itraconazole drug level should be measured 2 weeks after initiation ${ }^{9}$ and liver function should be monitored periodically.

In conclusion, it is essential to consider a broad differential diagnosis in immunosuppressed patients presenting with pulmonary disease. Aetiologies can include usual pathogens that infect immunocompetent hosts, opportunistic infections, and reactions with immunosuppressant therapy. Careful attention to the sensitivities, specificities and crossreactivities of tests for fungal diseases is extremely important. In immunocompromised patients from endemic regions, coinfection with Histoplasma and Pneumocystis is possible. ${ }^{10}$ It is important to conduct a thorough search for additional pathogens and avoid anchoring bias.

\section{Conflicts of interest}

The authors have no conflicts of interests to declare.

\section{Acknowledgements}

Written consent was obtained from the patient to publish the clinical details and images in this article. 
Table 2. ATS recommendations for management of histoplasmosis ${ }^{9}$

\section{Clinical situation}

\section{Immunocompetent hosts}

Asymptomatic pulmonary nodules

Asymptomatic broncholithiasis

Broncholithiasis causing post-obstructive pneumonia, haemoptysis, fistula or airway compromise

Fibrosing mediastinitis

Symptomatic mild pulmonary disease

Persistently symptomatic mild or moderate pulmonary disease

Severe/life-threatening pulmonary disease

\section{Immunocompromised hosts}

Mild to moderate pulmonary disease or progressive/ disseminated disease

Moderate to severe pulmonary disease or progressive/disseminated disease requiring hospitalisation

Chronic pulmonary disease or cavitation

Patients with AIDS who remain immunosuppressed, on lifelong maintenance therapy

ATS = American Thoracic Society; BID = two times a day; TID = three times a day

*In patients unable to tolerate itraconazole, alternatives include fluconazole and ketoconazole.

\section{References}

1 Daïen CI, Monnier A, Claudepierre P et al. Sarcoid-like granulomatosis in patients treated with tumor necrosis factor blockers: 10 cases. Rheumatology 2009;48:883-6.

2 Kanne JP, Yandow DR, Meyer CA. Pneumocystis jiroveci pneumonia: high-resolution CT findings in patients with and without HIV infection. AJR Am J Roentgenol. 2012;198:W555-61.

3 Kennedy CA, Goetz MB. Atypical roentgenographic manifestations of Pneumocystis carinii pneumonia. Arch Intern Med 1992;152:1390-8.

4 Azoulay E, Bergeron A, Chevret S et al. Polymerase chain reaction for diagnosing pneumocystis pneumonia in non-HIV immunocompromised patients with pulmonary infiltrates. Chest 2009;135:655-61.

5 Hartel PH, Shilo K, Klassen-Fischer M et al. Granulomatous reaction to pneumocystis jirovecii: clinicopathologic review of 20 cases. Am J Surg Pathol 2010;34:730-4.

6 Wheat LJ, Goldman M, Knox K. Cryptococcosis and the endemic mycoses. In: Fishman AP, Elias JA, Fishman JA et al (eds). Fishman's pulmonary diseases and disorders, 4 th edn. New York: McGraw-Hill, 2008:2327-49.
7 Kauffman CA. Histoplasmosis: a clinical and laboratory update. Clin Microbiol Rev 2007;20:115-32.

8 Limper AH, Knox KS, Sarosi GA et al. An official American Thoracic Society statement: treatment of fungal infections in adult pulmonary and critical care patients. Am J Respir Crit Care Med 2011;183:96-128.

9 Wheat LJ, Freifeld AG, Kleiman MB et al. Clinical practice guidelines for the management of patients with histoplasmosis: 2007 update by the Infectious Diseases Society of America. Clin Infect Dis 2007;45:807-25.

10 Baughman RP, Dohn MN, Frame PT. The continuing utility of bronchoalveolar lavage to diagnose opportunistic infection in AIDS patients. Am J Med 1994;97:515-22.

Address for correspondence: Dr Sumedh S Hoskote, 200 First Street SW, Rochester, MN 55905, USA.

Email: hoskote.sumedh@mayo.edu 